Background Parental physical activity (PA) is frequently investigated as a potential correlate of preschool-aged children's PA, yet there is little information about how the association between parent-child PA changes during the transition to formal schooling. We aimed to determine the association between objectively measured maternal and 6-year-old children's PA, exploring how this association differs by demographic and temporal factors; and 2) identify how this association changes during the transition to school (from 4-6 years).

Methods Data were from the UK Southampton Women's Survey. PA levels of 530 6-year-olds and their mothers were measured concurrently using accelerometry for up to 7 days. Two-level mixed-effects linear regression was used to model the association between maternal-child PA at age 6 [average activity intensity (ACC); minutes spent sedentary (SED); and in moderate and vigorous PA (MVPA)]. Interactions with demographic and temporal factors, and how the association differed across the day (morning (6-9am); school (9am-3pm) and evening $(3-11 \mathrm{pm})$ ), were tested. Change in the association between maternal-child PA (at age 4 and 6, n=170) was also assessed.

Results At age 6, mother-child daily PA were positively associated at all activity intensities: ACC: $\beta=0.24$ [95\% CI: 0.19 , $0.30]$ counts per minute; SED: 0.23 [0.20, 0.26] minutes/ hour; MVPA: 0.53 [0.43,0.64] minutes/hour. The association was stronger between mother-child PA at all intensities at the weekend (vs. weekdays: ACC: $\beta_{\text {interaction }}=0.16$ [95\% CI: 0.06 , $0.25]$ counts per minute; SED: 0.07 [0.02,0.12] minutes; MVPA: 0.44 [0.24,0.64] minutes). For SED, the mother-child association was stronger for children with older siblings (vs. none); for MVPA, the relationship was stronger for those who had both younger and older siblings (vs. none). Longitudinally, the mother-child association did not differ with age for SED and light PA (LPA); mother-child ACC and MVPA were significantly weaker at age 6 compared with age 4 (difference in ACC: $-0.23[-0.37,-0.10]$, MVPA: $-0.16[-0.31,-0.00])$. This difference was driven by a weaker relationship in the mornings and during the school day (9-3 pm).

Discussion Maternal-child PA levels are positively associated at age 6, with stronger associations at weekends, and in those with siblings in the home. From age 4 to 6 years, the mother-child ACC and MVPA association weakened. This may reflect decreasing co-participation with age, as children gain independence/engage in more structured PA at school. Different intervention foci may be needed before and after the transition to school, but family-based PA remains an important element of children's overall PA.

\section{OP68 PHYSICAL ACTIVITY TRAJECTORIES AND PREDICTORS DURING THE TRANSITION TO OLD AGE}

1,2DAA Aggio*, "EP Papachristou, ${ }^{1} \mathrm{OP}$ Papacosta, ${ }^{1} \mathrm{LL}$ Lennon, ${ }^{1} \mathrm{SA}$ Ash, ${ }^{3} \mathrm{PHW}$ Whincup, 1,2 $\mathrm{SGW}$ Wannamethee, ${ }^{1,2}$ BJJ Jefferis. 'UCL Department of Primary Care and Population Health, UCL, London, UK; ${ }^{2}$ Physical Activity Research Group, UCL, London, UK; ${ }^{3}$ Population Health Research Institute, St George's University of London, London, UK

10.1136/jech-2017-SSMAbstracts.67

Background Maintaining physical activity during later life is associated with optimal health; however, research on the longterm trajectories of physical activity into old age and their predictors has been limited. This study aimed to identify distinct 20 year trajectories of physical activity spanning from midlife to old age and predictors of physical activity trajectories.

Methods Participants were men drawn from the British Regional Heart Study, a prospective cohort study, involving 7735 men recruited from Primary Care Practices in 1978-80. Men were followed up after 12, 16 and 20 years, reporting physical activity levels (walking, cycling, recreational activity and sport/exercise), health status and socio-demographic characteristics. Group-based trajectory modelling was applied to identify distinct trajectories of physical activity and to examine the effects of predictor variables on trajectories. Predictors of trajectory group membership were examined using multinomial logistic regression. The effects of developing cardiovascular disease and changing employment status on trajectories were estimated for each trajectory group.

Results 7658 men (mean baseline age 50.2 \pm 5.8 years) providing valid questionnaire and physical activity data (of which $78 \%$ provided activity data at $\geq 2$ surveys) were included in analyses. Three distinct trajectories of physical activity emerged: low decreasing (34.1\%), low stable (45.8\%) and moderate increasing (20.1\%). Membership of the moderate increasing trajectory group was predicted by being married, having children, drinking alcohol and eating breakfast. Men with manual occupations, men residing in regions other than the South, men who were overweight/obese, men with doctordiagnosed health conditions and men who smoked were less likely to be in the moderate increasing trajectory group. Being employed was associated with an increase in physical activity in the low decreasing group $(\beta 0.43, p<0.001)$ but a decrease in the low stable $(\beta-0.22, \mathrm{p}<0.001)$ and moderate increasing groups $(\beta-0.84, \mathrm{p}<0.001)$. Development of cardiovascularrelated conditions was associated with a decline in physical activity in the low decreasing $(\beta-0.42, \mathrm{p}<0.001)$ and low stable groups $(\beta-0.13, \mathrm{p}<0.05)$ but was not associated with any change in the moderate increasing group $(\beta-0.06$, $\mathrm{p}=0.34)$.

Conclusion This study highlights the heterogeneity in physical activity levels over time in older adults and that activity levels in midlife are likely to dictate trajectories into old age. Efforts to promote physical activity in later life may need to focus attention earlier in the lifecourse. The effects of retirement and cardiovascular disease on physical activity may depend on prior activity trajectories. Thus, different strategies may be needed in these groups.

\section{Ageing 2}

\section{OP69 CIRCULATING N-3 POLYUNSATURATED-FATTY ACIDS
AND THE MAINTENANCE OF HEALTHY AGEING IN
OLDER ADULTS, THE CARDIOVASCULAR HEALTH STUDY}

${ }^{1} \mathrm{HT}$ Lai* ${ }^{2} \mathrm{MC}$ de Oliveira Otto, ${ }^{3} \mathrm{RN}$ Lemaitre, ${ }^{3} \mathrm{~B}$ McKnight, ${ }^{4} \mathrm{PHM}$ Chaves, ${ }^{5} \mathrm{MC}$ Odden ${ }^{6} \mathrm{AB}$ Newman, ${ }^{7}$ DS Siscovick, ${ }^{1} \mathrm{D}$ Mozaffarian. ${ }^{1}$ Friedman School of Nutrition Science and Policy, Tufts University, Boston, Massachusetts, USA; ${ }^{2}$ Department of Epidemiology, University of Texas Health Science Centre at Houston, Houston, Texas, USA; ${ }^{3}$ Cardiovascular Health Research Unit, University of Washington, Seattle, Washington, USA; ${ }^{4}$ Benjamin Leon Centre for Geriatric Research and Education, Florida International University Hebert Wertheim College of Medicine, Miami, Florida, USA; ${ }^{5}$ School of Biological and Population Health Sciences, Oregon State University, Corvallis, Oregon, USA; ${ }^{6}$ Department of Epidemiology, University of Pittsburgh Graduate School of Public Health, Pittsburgh, Pennsylvania, USA; ${ }^{7}$ New York Academy of Medicine, New York City, New York, USA

10.1136/jech-2017-SSMAbstracts.68 